\section{Enterobakterien als Reisesouvenir}

Infektionen als Urlaubsmitbringsel von Reisen in weniger entwickelte Länder sind nicht selten. Das gilt auch für ESBL-bildende Enterobakterien. Laut einer prospektiven Studie hatte jeder dritte zuvor nicht infizierte Teilnehmer (34,3\%) die resistenten Keime von seiner Reise mitgebracht. Die höchste Rate wurde bei denen festgestellt, deren Reiseziel in einem Land im Süden Asiens lag. Sie betrug 75\%. Der Anteil der Infizierten mit Reiseziel Zentral- und Ostasien lag bei knapp $49 \%$ und mit Ziel Westasien oder Nordafrika bei jeweils etwa $42 \%$. Das Risiko, sich in Südafrika, Nordamerika, Europa oder Ozeanien zu infizieren, war mit $6 \%$ am geringsten.

Arcilla M S et al. Lancet Infect Dis 2016 (online first)

\section{Rektum-Ca.: Länger leben mit Radiatio}

Einer Datenanalyse mit mehr als $21.700 \mathrm{~Pa}$ tienten zufolge verlängert die neoadjuvante Bestrahlung von Patienten mit Rektumkarzinom das Leben. 21.433 Patienten erhielten - wie es der Standard ist - eine neoadjuvante Chemoradiotherapie, 274 Patienten nur eine Mehrfachchemokombitherapie. Danach erfolgte eine Operation. Die Auswertung der Daten ergab, dass die Patienten mit einer Monochemotherapie und der Bestrahlung davon einen größeren Nutzen hatten als die Patienten der Vergleichsgruppe ohne Bestrahlung. Nach fünf Jahren lebten von den bestrahlten $\mathrm{Pa}$ tienten noch $75 \%$, in der Vergleichsgruppe noch $67,2 \%$ - ein signifikanter Unterschied.

Cassidy RJ et al. Cancer 2016 (online first)

\title{
Hoher BMI und Typ-2-Diabetes sind mit Leberkrebs assoziiert
}

Die Auswertung der Daten von mehr als 1,5 Millionen US-Studienteilnehmern bestätigt, dass bei Menschen mit hohem BMI, großem Taillenumfang oder Typ2-Diabetes die Wahrscheinlichkeit, an einem hepatozellulären Karzinom zu erkranken, erhöht ist. Mithilfe der gängigsten Regressionsanalyse „Cox Proportional Hazards Model“ bestimmten Dr. Peter T. Campbell, Atlanta, und Kollegen die Assoziationen der Faktoren BMI, Taillenumfang und Typ-2-Diabetes mit der Entwicklung eines hepatozellulären Karzinoms. Im Vergleich zu einem normalen BMI, also zwischen 18,5 und $25 \mathrm{~kg} / \mathrm{m}^{2}$, ging ein BMI von 25 bis $29,5 \mathrm{~kg} / \mathrm{m}^{2}$ mit einem um $21 \%$, ein BMI $\geq$ $40 \mathrm{~kg} / \mathrm{m}^{2}$ sogar mit einem um $116 \%$ erhöhten Leberkrebsrisiko einher. Die Wahrscheinlichkeit für Typ-2-Diabetiker, an Leberkrebs zu erkranken, war fast um das Doppelte erhöht.

Campbell PT et al. Cancer Res 2016; 76 (20):6076-6083

\section{Pankreatitisgefahr steigt schon bei leicht erhöhten Triglyzeriden}

Schon Serumtriglyzeride ab $177 \mathrm{mg} / \mathrm{dl}$ ( $2 \mathrm{mmol} / \mathrm{l}$ ) im nicht nüchternen Zustand sind dänischen Ärzten zufolge mit „einem hohen, relativen Risiko“ einer akuten Pankreatitis assoziiert. Das relative Erkrankungsrisiko steigt in Abhängigkeit von der Triglyzeridkonzentration sogar stärker als das Herzinfarktrisiko, wie Simon B. Pedersen aus Herlev und Kollegen berichten. Die Erkenntnisse beruhen auf der Analyse von prospektiv erhobenen Daten aus zwei bevölkerungsbasierten Kohorten, der Copen- hagen General Population Study und der Copenhagen Heart Study. Von zusammen 116.550 Teilnehmern (medianes Alter 57 Jahre) hatten während der Beobachtungszeit von median 6,7 Jahren 434 eine akute Entzündung der Bauchspeicheldrüse und 3942 einen Herzinfarkt erlitten. Pro Zunahme der Serumtriglyzeride um $89 \mathrm{mg} / \mathrm{dl}$ ( $1 \mathrm{mmol} / \mathrm{l})$ erhöhte sich das Risiko einer akuten Pankreatitis um 17\%.

Pedersen SB et al. JAMA Intern Med 2016 (online first)

\section{Alarm im Darm!}

Wenn Patienten über Bauchschmerzen, Durchfall oder Verstopfung klagen, leiden sie möglicherweise an einem Reizdarmsyndrom. Da jedoch die Symptome so vielfältig wie individuell sind, kommt einer gewissenhaften (Ausschluss-)diagnostik eine wichtige Rolle zu. Dazu gehört auch die einmalige Koloskopie, um Krankheiten wie das kolorektale Karzinom sicher auszuschließen. Wie Sie dem Darm wieder zu mehr Schwung verhelfen, lesen Sie neben vielen weiteren spannenden Themen im Schwerpunkt "Gastroenterologie".

Claudia Daniels

Redakteurin

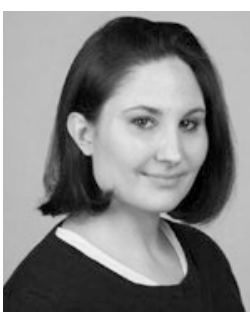

\section{Schlafmangel} weckt den Appetit

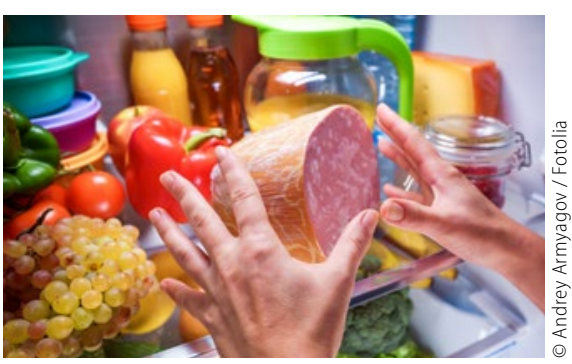

Wer nicht genug schläft, bekommt schneller Heißhungerattacken.

Ärzte haben versucht, den Zusammenhang zwischen Schlafmangel und Gewichtszunahme in einer Metaanalyse zu quantifizieren: Berücksichtigt wurden 17 Studien mit 496 Teilnehmern im Alter zwischen 18 und 50 Jahren. Die maximale Dauer einer Testphase lag bei zwei Wochen. Als partieller Schlafentzug galt ein Nachtschlaf von weniger als vier bis $\mathrm{zu}$ fünfeinhalb Stunden. In die Metaanalyse zur Energiezufuhr konnten zehn Studien einbezogen werden. Dabei zeigte sich ein hochsignifikanter Unterschied zwischen den 185 Teilnehmern mit und den 161 ohne Schlafrestriktion: Der Schlafmangel schlug mit zusätzlichen $385 \mathrm{kcal}$ zu Buche.

Al Khatib Het al. European Journal of Clinical Nutrition 2016 (online first) 